\title{
Clinical Efficacy of Symptomatic Manifestations in Prediction of Pediatric Obstructive Sleep Apnea
}

\author{
Woo Hyun Lee, MD, Seong II Kang, MD, Jeong-Whun Kim, MD \\ Department of Otorhinolaryngology, Seoul National University Bundang Hospital, Seoul National University College of Medicine, Seongnam, Korea
}

\begin{abstract}
Background and Objective This study was aimed to identify clinical efficacy of subjective symptoms in prediction of obstructive sleep apnea (OSA) in children.

Methods Medical records of children under age of 12 years who underwent attended full night polysomnography were reviewed. Questionnaires in the Korean version of OSA-18 were answered by all of the patients. Among the questionnaires, the scores of snoring, apnea and mouth breathing were summated to analyze the correlation between subjective symptoms and apnea hypopnea index (AHI). The hypertrophy of the tonsils and adenoids were graded from 1 to 4 .

Results A total of 68 patients (45 males and 23 females) were included in this study. The average age of patients was 6.9 years. The average score of snoring, apnea and mouth breathing was 5.1, 3.7 and 5.1, respectively. The average grade of tonsil and adenoid hypertrophy was 2.9 and 2.5, respectively. While the Korean version of OSA-18 score and AHI did not show a significant correlation $(p=0.222)$, the sum of snoring, apnea and mouth breathing score showed a significant correlation with AHI $(\mathrm{p}=0.019)$. A composite score including snoring, apnea, mouth breathing, and adenotonsillar hypertrophy was also correlated with AHI ( $\mathrm{p}=0.035)$. However, adenotonsillar hypertrophy was not correlated with AHI.
\end{abstract}

Conclusions The present study showed that OSA in children was better predicted by subjective symptoms, in particular, snoring, apnea and mouth breathing than adenotonsillar hypertrophy.

Sleep Med Res 2014;5(1):25-28

Key Words Tonsil, Adenoid, Snoring, Obstructive sleep apnea, Pediatric.

Received: December 1, 2014 Revised: December 10, 2014 Accepted: December 15, 2014 Correspondence Jeong-Whun Kim, MD Department of Otorhinolaryngology,

Seoul National University Bundang Hospital, Seoul National University College of Medicine, 82 Gumi-ro 173beon-gil, Bundang-gu, Seongnam 463-707, Korea

Tel +82-31-787-7405

Fax +82-31-787-4057

E-mail kimemail@snubh.org

\section{INTRODUCTION}

Obstructive sleep apnea (OSA) in children can also lead to several co-morbidities such as attention deficit, dentofacial abnormality, poor school performance, growth retardation, heart problem, hypertension, or enuresis. ${ }^{1,2}$ It was also reported that the children with OSA had high prevalence of neurobehavioral deficiency and it was significantly improved after adenotonsillectomy. ${ }^{3}$ Therefore, its early diagnosis and treatment has drawn attention from sleep physicians and parents. Hypertrophy of the tonsils and adenoids are the most common cause of OSA in children. ${ }^{4}$ The severity of OSA can be diagnosed by full night polysomnography and American Academy of Pediatrics recommends nocturnal polysomnography as a gold standard test for diagnosis of OSA in children before adenotonsillectomy. ${ }^{5}$ However, most of the patients undergo adenotonsillectomy without sleep study results when they are diagnosed as having hypertrophic tonsils or adenoids because surgeons contemplate that the night time symptoms will be much improved after adenotonsillectomy in general. However, it is still difficult to predict the severity of OSA and explain it to parents simply on the basis of adenotonsillar hypertrophy. The main manifestations of OSA in children are snoring, pause of breathing, apnea, and mouth breathing. The present study is aimed to identify whether a minimal combination of questionnaires will be useful in prediction of OSA in children. 


\section{METHODS}

\section{Patient Selection}

Children with age of 12 years or less who visited the sleep clinic with symptoms of snoring, breathing difficulties or mouth breathing during sleep between September 2010 and June 2012 were retrospectively enrolled. Children with congenital disorders, Down's syndrome, neuromuscular diseases and chronic lung diseases were excluded. All of them underwent attended full night polysomnography and their medical records were reviewed. They underwent endoscopic nasal and oropharyngeal examination and lateral cephalometry was performed. The parents of the children were asked to answer questionnaires in the Korean version of OSA-18. This study was approved by the Institutional Review Board.

\section{Attended Full Night Polysomnography}

Polysomnography (Embla ${ }^{\mathrm{TM}}$ N 7000, Embla, Reykjavik, Iceland) were performed with standard electrodes and sensors. Electroencephalography electrodes were applied at C4/A1, C3/ A2, O1/A2 and O2/A1 and two electrooculography electrodes were applied. Electromyography electrodes were applied at the submentalis muscle and anterior tibialis muscle. A strain gage was used for recording chest and abdominal respiratory movement and a nasal pressure cannula was used to record airflow. Oxygen saturation was measured using a pulse oxymeter applied to the index finger. Based on the criteria of Rechtschaffen and Kales, sleep was scored for every 30-second epoch of the polysomnogram. Apnea was defined as complete cessation of airflow for at least 2 breaths. Hypopnea was defined as a substantial reduction in airflow (>50\%) for at least 2 breaths or a moderate reduction in airflow for at least 2 breaths associated with electroencephalographic arousal or oxygen desaturation ( $\geq 3 \%$ ). The apnea hypopnea index (AHI) was defined as the total number of apneas and hypopneas per hour of sleep and the oxygen desaturation index was calculated as the number of oxygen desaturations $(\geq 4 \%)$ per hour of sleep. ${ }^{6,7}$

\section{Grading of Adenotonsillar Hypertrophy}

Grading of the adenoids and palatine tonsils was performed as previously performed. ${ }^{8}$ In brief, the adenoids were graded using the lateral cephalometry. The ratio of the adenoids to nasopharynx was defined as the ratio between the adenoid thickness and nasopharyngeal width, and it was designated as $\mathrm{A} / \mathrm{N}$ ratio. The grade of adenoid thickness was defined according to the $\mathrm{A} / \mathrm{N}$ ratio: grade 1 denotes $0 \%$ to $25 \%$, grade 2 denotes $25 \%$ to $50 \%$, grade 3 denotes $50 \%$ to $75 \%$, and grade 4 denotes $75 \%$ to $100 \%$. The tonsils were graded from 1 to 4 according to the grading system of tonsil size in Friedman staging system, which has strong correlation with objective tonsil volume., ${ }^{9,10}$

\section{Korean Version of OSA-18}

Parents of the patients were asked to sleep questionnaires of the Korean version of OSA-18. ${ }^{11}$ The OSA-18 consists of 18 items in five domains (sleep disturbance, physical suffering, emotional distress, daytime problems, and caregiver concerns). A scoring system was used ranging from 1 (none of the time) to 7 (all of the time) to grade the relative severity of the problem addressed in each item. The total score of OSA-18 was from 18 to 126 . Out of the items in the questionnaire, the sum of snoring, breath pause at night and mouth breathing score was calculated.

\section{Statistical Analyses}

Correlations analyses were performed to identify the relationship between AHI and subjective symptoms, adenotonsillar hypertrophy or a composite parameter including both of subjective symptoms and adenotonsillar hypertrophy. Receiver operating characteristic (ROC) curve analyses were performed to identify cutoff values of parameters to predict OSA in children. A value of $\mathrm{p}<0.05$ was considered statistically significant.

\section{RESULTS}

A total of 68 patients ( 45 males and 23 females) were included in this study. The average age of patients was 6.9 years. The average $\mathrm{z}$-score of body mass index was $-0.19 \pm 1.39$. The average of tonsil and adenoid grade was $2.9 \pm 0.9$ and $2.5 \pm 1.1$ and the average sum of tonsil and adenoid grade was $5.3 \pm 1.6$. The average score of Korean version of OSA-18 was $64.5 \pm 21.7$. The score of snoring, apnea and mouth breathing was $5.1 \pm 1.6$, $3.7 \pm 1.7$, and $5.1 \pm 1.7$, respectively. The average sum of snoring, apnea and mouth breathing score was $13.9 \pm 4.1$. The average composite score of subjective symptoms (snoring, apnea and mouth breathing) and adenotonsillar hypertrophy was $19.1 \pm$ 4.7. The median AHI was 3.7.

\section{Correlation between AHI and Adenotonsillar Hypertrophy}

There was no significant correlation between AHI and tonsil grade $(\mathrm{p}=0.636)$ or adenoid grade $(\mathrm{p}=0.603)$. AHI also showed no correlation with the sum of tonsil grade and adenoid grade $(\mathrm{p}=0.362)$.

\section{Correlation between AHI and Subjective Symptoms}

There was no correlation between AHI and the total score of Korean version of OSA-18 ( $p=0.222)$. On the other hand, AHI had a significant correlation with the sum of snoring, apnea and mouth breathing score $(\mathrm{p}=0.019)$. AHI also has a significant correlation with the composite score including the three subjective symptoms and adenotonsillar hypertrophy $(\mathrm{p}=0.035)$. However, when we put the same weight on the symptoms (50\%) 
and adenotonsillar hypertrophy (50\%), there was no significant correlation between AHI and the composite score. $(\mathrm{p}=0.114)$ (Table 1).

\section{ROC Curve Analyses}

Receiver operating characteristic curve analyses were performed when pediatric OSA was diagnosed with AHI > 1.0. The cutoff point for the sum of snoring, apnea and mouth breathing score to predict OSA in children (AHI $\geq 1$ ) was 13.5 (sensitivity $=56.0 \%$, specificity $=62.7 \%$, and area under curve $=0.618)$. The cutoff point for the composite score including adenotonsillar size grade in addition to the 3 symptoms was 17.5 (sensitivity $=66.0 \%$, specificity $=62.5 \%$, and area under curve $=0.620)($ Fig. 1$)$.

\section{DISCUSSION}

The present study revealed that subjective symptoms were more significantly correlated with AHI than the size grade of the palatine tonsils or adenoids. In a practical clinical setting, even though clinicians know that polysomnography is a gold

Table 1. Relationship between $\mathrm{AHI}$ and adenotonsillar hypertrophy or subjective symptoms

\begin{tabular}{lcc}
\hline & $\begin{array}{c}\text { Correlation } \\
\text { coefficient }\end{array}$ & p-value \\
\hline Tonsil size grade & 0.058 & 0.636 \\
Adenoid size grade & 0.064 & 0.603 \\
Tonsil \& adenoid size grade & 0.112 & 0.362 \\
Korean version of OSA-18 & 0.188 & 0.222 \\
Sum of snoring, apnea \& mouth & 0.307 & 0.019 \\
$\quad$ breathing score & & \\
Composite score of snoring, apnea, & 0.277 & 0.035 \\
$\quad$ mouth breathing \& adenotonsillar & & \\
hypertrophy grade & & \\
\hline AHI: apnea hypopnea index, OSA: obstructive sleep apnea.
\end{tabular}

standard for diagnosis of OSA and for decision-making of adenotonsillectomy in children, it is not general for both clinicians and parents to reach an agreement on conduction of polysomnography because of the following reasons. Firstly, polysomnography has some limitations such as high cost and uncomfortable sleeping environment for children. Secondly, most of the children with adenotonsillar hypertrophy have much improvement after surgical treatment. Nonetheless, it may not be recommendable for sleep physicians and surgeons not to predict the severity of OSA before performing adenotonsillectomy. Even parents want to know whether their children will be diagnosed as having OSA and be informed of it by physicians in a hospital even if they are reluctant to pay for polysomnography.

Therefore, it is necessary to predict the presence of OSA using several objective findings and subjective symptoms from children. In general, the size of the tonsils and adenoids seem to be the most commonly used for determination of surgical indication and for prediction of OSA. There have been controversies on the predictability of adenotonsillar size for OSA. Some studies showed that adenotonsillar size did not predict the severity of $\mathrm{AHI}^{12,13}$ while another study revealed that tonsil weight can be a predictor of AHI. ${ }^{14}$ On the other hand, OSA-18 has been most commonly used as a survey to predict OSA in children. ${ }^{11}$ OSA-18 has been shown to be effective as a tool to compare the quality of life before and after adenotonsillectomy. ${ }^{15,16}$ However, some clinical researches suggested that OSA-18 had poor validity in detecting and predicting pediatric OSA. ${ }^{17,18}$ The OSA18 questionnaire consists of 18 items including psychological and parental parameters as well as physical parameters. It also takes some time to fill up all the items. In the present study, we tried to identify the efficacy of a minimal combination of questionnaires in detecting pediatric OSA. Snoring, pause of breathing and mouth breathing are the 3 most common manifestations of children who visit pediatric sleep clinic. Therefore, we developed a new summated score combining snoring, apnea and mouth breathing score. Furthermore, we analyzed to iden-
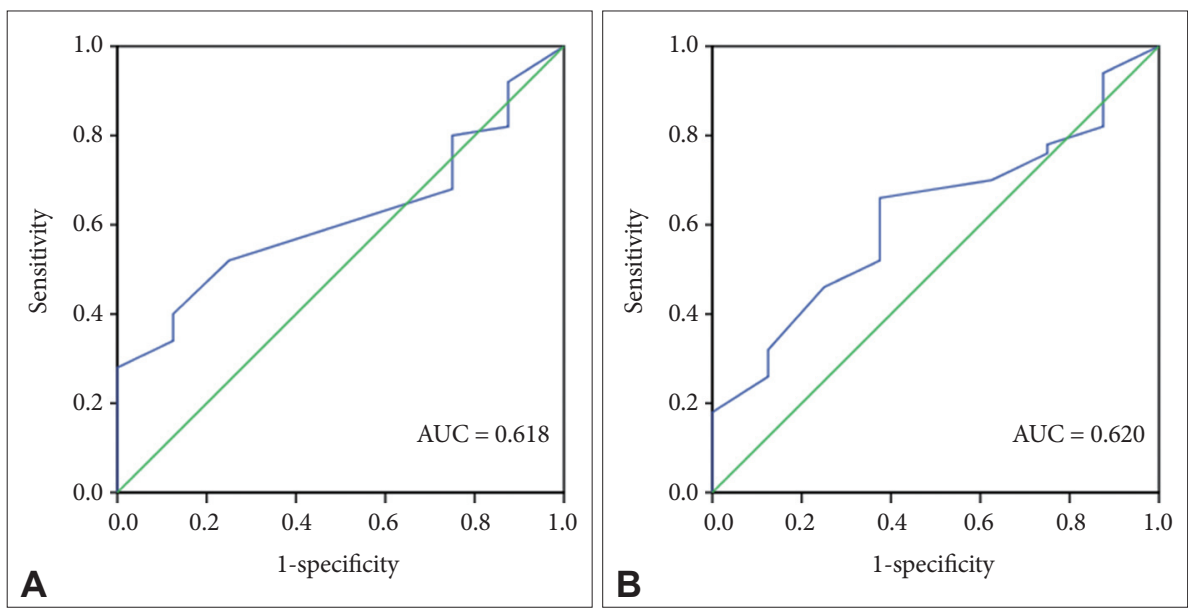

Fig. 1. Receiver operating characteristic $(R O C)$ curve. A: The area under the ROC curve of the sum of snoring, apnea and mouth breathing score for apnea hypopnea index was 0.618 (sensitivity = $56.0 \%$, specificity $=62.7 \%)$. B: The area under the ROC curve of composite score of snoring, apnea, mouth breathing \& adenotonsillar hypertrophy grade for apnea hypopnea index was 0.620 (sensitivity = $66.0 \%$, specificity $=62.5 \%$ ). AUC: area under curve. 
tify whether addition of adenotonsillar size grade to the symptom score will show a synergistic effect on detection of pediatric OSA. We found that only the size of the tonsils and adenoids was not useful and synergistic in detection of pediatric OSA. On the other hand, the minimal combination of major symptomatic manifestations was significantly correlated with AHI while the OSA-18 did not show significant correlation with AHI.

In conclusion, the present study demonstrated that AHI of OSA in children were better correlated with subjective symptoms, in particular, snoring, apnea and mouth breathing than the anatomical size grade of adenotonsillar hypertrophy. Instead of the whole OSA-18 questionnaires, its breathing-associated key items may be effectively used in prediction of OSA. Considering that the present study did not include a large number of children, a future study is warranted to validate the efficacy of the minimal combination of symptom scores in detection and prediction of pediatric OSA.

\section{Conflicts of Interest}

The authors have no financial conflicts of interest.

\section{REFERENCES}

1. Brouillette RT, Fernbach SK, Hunt CE. Obstructive sleep apnea in infants and children. J Pediatr 1982;100:31-40.

2. Marcus CL. Sleep-disordered breathing in children. Am J Respir Crit Care Med 2001;164:16-30.

3. Chervin RD, Weatherly RA, Garetz SL, Ruzicka DL, Giordani BJ, Hodges EK, et al. Pediatric sleep questionnaire: prediction of sleep apnea and outcomes. Arch Otolaryngol Head Neck Surg 2007;133:216-22.

4. De Serres LM, Derkay C, Sie K, Biavati M, Jones J, Tunkel D, et al. Impact of adenotonsillectomy on quality of life in children with obstructive sleep disorders. Arch Otolaryngol Head Neck Surg 2002;128:489-96.

5. Marcus CL, Brooks LJ, Draper KA, Gozal D, Halbower AC, Jones J, et al. Diagnosis and management of childhood obstructive sleep apnea syndrome. Pediatrics 2012;130:e714-55.

6. Iber C, American Academy of Sleep Medicine. The AASM manual for the scoring of sleep and associated events: rules, terminology and technical specifications. Westchester, IL: American Academy of Sleep Medicine 2007.

7. Witmans MB, Keens TG, Davidson Ward SL, Marcus CL. Obstructive hypopneas in children and adolescents: normal values. Am J Respir Crit Care Med 2003;168:1540.

8. Kim SY, Lee WH, Rhee CS, Lee CH, Kim JW. Regrowth of the adenoids after coblation adenoidectomy: cephalometric analysis. Laryngoscope 2013;123:2567-72.

9. Friedman M, Ibrahim H, Bass L. Clinical staging for sleep-disordered breathing. Otolaryngol Head Neck Surg 2002;127:13-21.

10. Cahali MB, Soares CF, Dantas DA, Formigoni GG. Tonsil volume, tonsil grade and obstructive sleep apnea: is there any meaningful correlation? Clinics (Sao Paulo) 2011;66:1347-52.

11. Franco RA Jr, Rosenfeld RM, Rao M. First place--resident clinical science award 1999. Quality of life for children with obstructive sleep apnea. Otolaryngol Head Neck Surg 2000;123(1 Pt 1):9-16.

12. Brooks LJ, Stephens BM, Bacevice AM. Adenoid size is related to severity but not the number of episodes of obstructive apnea in children. $J$ Pediatr 1998;132:682-6.

13. Hwang SH, Guilleminault C, Park CS, Kim TW, Hong SC. Usefulness of adenotonsillar size for prediction of severity of obstructive sleep apnea and flow limitation. Otolaryngol Head Neck Surg 2013;149:326-34.

14. Howard NS, Brietzke SE. Pediatric tonsil size: objective vs subjective measurements correlated to overnight polysomnogram. Otolaryngol Head Neck Surg 2009;140:675-81.

15. Baldassari CM, Mitchell RB, Schubert C, Rudnick EF. Pediatric obstructive sleep apnea and quality of life: a meta-analysis. Otolaryngol Head Neck Surg 2008;138:265-73.

16. Hasukic B. OSA-18 survey in evaluation of sleep-disordered breathing in children with adenotonsillar hypertrophy. Med Arch 2013;67:111-4.

17. Borgström A, Nerfeldt P, Friberg D. Questionnaire OSA-18 has poor validity compared to polysomnography in pediatric obstructive sleep apnea. Int J Pediatr Otorhinolaryngol 2013;77:1864-8.

18. Constantin E, Tewfik TL, Brouillette RT. Can the OSA-18 quality-of-life questionnaire detect obstructive sleep apnea in children? Pediatrics 2010; 125:e162-8. 